\title{
GRUPOS FUNCIONALES ALIMENTICIOS DE MACROINVERTEBRADOS ACUÁTICOS EN UN ARROYO TROPICAL (COLOMBIA)
}

\section{FUNCTIONAL FEEDING GROUPS OF AQUATIC MACROINVERTEBRATES IN A TROPICAL STREAM (COLOMBIA)}

\author{
Ángela Motta Díaz ${ }^{1}$, Laura Ortega Corredor ${ }^{2}$, Yeina Niño Fernández ${ }^{3}$, Nelson Aranguren Riaño ${ }^{4}$
}

\begin{abstract}
${ }^{1}$ Biólogo. U. Pedagógica y Tecnología de Colombia, Grupo de investigación Unidad de Ecología en Sistemas Acuáticos, Avenida Central del Norte, Vía Paipa, Tunja-Boyacá, Colombia, e-mail: angelitaconejon@gmail.com; ${ }^{2}$ Biólogo. U. Pedagógica y Tecnología de Colombia, Grupo de investigación Unidad de Ecología en Sistemas Acuáticos, Tunja-Boyacá, Colombia, e-mail: laurandreaoc@gmail.com; ${ }^{3}$ Biólogo. U. Pedagógica y Tecnología de Colombia, Tunja-Boyacá, Colombia, e-mail: rijoal1122@yahoo.es; ${ }^{4}$ Ph.D. en Ciencias Biológicas. U. Pedagógica y Tecnología de Colombia, Facultad de Ciencias Básicas, Escuela de Biología, Grupo de investigación Unidad de Ecología en Sistemas Acuáticos, Tunja- Boyacá, Colombia, e-mail: nelson.aranguren@uptc.edu.co
\end{abstract}

Rev. U.D.C.A Act. \& Div. Cient. 19(2): 425-433, Julio-Diciembre, 2016

\section{RESUMEN}

Los macroinvertebrados son un importante componente biológico de los ríos, ya que están vinculados, activamente, en el flujo de materia y energía. El objetivo del estudio fue determinar la estructura y los grupos funcionales alimenticios de los macroinvertebrados, en la quebrada La Chapa (Departamento de Boyacá). En tres tramos de la quebrada, en las épocas de lluvia y sequía, se midieron características físicas y químicas. Mediante red Surber, se obtuvieron muestras en cada tramo, para estimar la abundancia y la biomasa de macroinvertebrados. La asignación de los grupos funcionales alimenticios por taxón, se hizo a partir de fuentes secundarias. En el análisis de componentes principales, las variables físicas y químicas se ordenaron según la temporalidad; la conductividad eléctrica, la dureza y la alcalinidad fueron las variables con mayor peso en el análisis. Respecto a los macroinvertebrados, se hallaron 35 taxones, con mayor riqueza en efemerópteros y dípteros, con 8 y 7 taxones, respectivamente. En época de lluvias predominaron, en abundancia, los organismos colectores-recolectores y colectoresfiltradores, como Thraulodes sp. y Simulium sp., mientras que en época seca fueron los raspadores, como Helycopsyche sp. En cuanto a la biomasa, en la época de lluvia predominaron los perforadores-carnívoros, como Limnocoris sp., mientras en la época seca fueron los raspadores, como Hydrobiidae. La variación temporal en los grupos funcionales, se puede asociar con los cambios en las condiciones del hábitat, determinados por la expresión diferencial del cau- dal. Se confirma que los cambios temporales asociados a la estacionalidad climática e hidráulica son importantes en la estructuración de la comunidad de macroinvertebrados, en los sistemas loticos andinos.

Palabras clave: Macroinvertebrados, estructura trófica, ríos de cabecera, Andes,

\section{SUMMARY}

Aquatic macroinvertebrates are an important biological component of rivers because they contribute to the flow of matter and energy. The goal of this study was to determine the structure and functional feeding groups of macroinvertebrates in the stream La Chapa (Dpto. Boyacá). In three sections of the stream, during the rainy and dry seasons, we measured physical and chemical characteristics of the water and used a surber net to obtain macroinvertebrate samples. Next, all species were identified, abundance and biomass calculated, then assigned each taxon to a functional feeding group based on dietary preferences. In the principal component analysis the physical and chemical variables were organized according to seasonality, electric conductivity, hardness and alkalinity. Thirtyfive macroinvertebrate taxa were found, with a high abundance of mayflies and diptera, with 8 and 7 taxa respectively. During the rainy season the stream was dominated by collector-gatherer and collectorfilter organisms including Thraulodes sp. and Simulium sp., while in the dry season scrapers such as Helycopsyche sp. 
were more common. In terms of biomass, the rainy season was composed primarily of the driller-carnivore Limnocoris sp., while in the dry season the biomass of the scrapers such as Hydrobiidae increased. The temporal variation in the functional feeding groups that we observed is likely a consequence of changes in habitat conditions over time. In conclusion, our work supports the role for seasonal changes in structuring the phenology of macroinvertebrate communities in Andean lotic ecosystems.

Key words: Macroinvertebrates, trophic structure, headstreams, Andes.

\section{INTRODUCCIÓN}

Los ríos de montaña andinos presentan características topográficas, geológicas e hidráulicas únicas, dadas por los cambios de caudal, entre épocas (seca-lluvias), su ubicación en la región húmeda tropical y en montañas geológicamente jóvenes, con altas pendientes. Estas circunstancias condicionan procesos físicos, químicos, biológicos y metabólicos, tales como: el transporte de sedimentos, la concentración de iones y sólidos disueltos y el procesamiento de materia orgánica (Jacobsen, 2008). Cerca de estos sistemas, se encuentran asentamientos humanos, que afectan negativamente la calidad ecológica, con actividades agropecuarias, de minería, extracción de agua y vertimientos de desechos (García et al. 2015).

Según Wantzen et al. (2008), existe gran variedad de fuentes de materia orgánica en estos sistemas, aunque la principal oferta, se halla en forma de materia orgánica particulada fina (MOPF), resultante de la dinámica hidráulica del río, debido al fraccionamiento físico y biológico de la MOPG. También, se destaca, en menor grado, la materia particulada gruesa (MOPG), compuesta, principalmente, por hojas caídas y restos de animales (Sabater et al. 2009).

Los macroinvertebrados cumplen importantes funciones en la transformación de la materia orgánica fina y gruesa, por ende, son un componente fundamental en la dinámica y funcionamiento del río. Debido a esto, han desarrollado modificaciones morfológicas y fisiológicas, que les ayudan a la adaptación en el hábitat, como son las estrategias alimenticias, las cuales, se organizan en grupos funcionales (Cummins, 1974). En este sentido, existen cinco grupos funcionales alimenticios principales: trituradores, que se alimentan de MOPG; colectores-recolectores; colectores filtradores de MOPF; raspadores de biopelícula y depredadores, que consumen otros animales (Cummins, 1974). En quebradas de montaña, se observa variedad de oferta alimenticia, que se relaciona con la presencia de ciertos grupos funcionales; en general, en estos sistemas, se destacan los organismos raspadores y colectores-recolectores (Tomanova et al. 2006).
Los cambios temporales de la estructura y la función de los macroinvertebrados acuáticos en sistemas Neotropicales están influenciados por las variaciones climáticas y la incidencia de éstas sobre la dinámica de flujo; estas perturbaciones naturales desempeñan un papel importante en la estructuración de la comunidad acuática (Rios-Touma et al. 2011). En Colombia, se han desarrollado trabajos que aprovechan la información de composición y de función de las comunidades de macroinvertebrados en quebradas de montaña, como por ejemplo, los de Rodríguez-Barrios et al. (2011), Chará-Serna et al. (2010), Guzmán-Soto \& Tamarís-Turizo (2014), Rivera et al. (2013) y Tamarís-Turizo \& RodríguezBarrios (2015). Regularmente, estos autores sugieren que se deben realizar más estudios ecológicos, que aborden otros aspectos, como grupos funcionales y auto-ecología, que permitirían entender mejor los procesos de autorregulación en los sistemas acuáticos y la influencia de perturbaciones naturales o artificiales, sobre éstos. La información sobre aspectos funcionales y cuantitativos de las comunidades acuáticas permiten cualificar los sistemas de evaluación de la calidad ecológica de los ríos que utilizan, principalmente, la estructura de las comunidades, como criterio de indicación (Bonada et al. 2006).

Dada la importancia de los macroinvertebrados en el funcionamiento de los sistemas de montaña, nuestra hipótesis de que la expresión de los grupos funcionales está dada por la época climática y la calidad ecológica del sistema, el objetivo de este trabajo fue determinar la estructura y los grupos funcionales alimentarios de los macroinvertebrados acuáticos, en una quebrada alto-andina y el uso potencial de esta información, para evaluar la calidad ecológica del sistema.

\section{MATERIALES Y MÉTODOS}

Área de estudio: El presente estudio, se desarrolló en la quebrada La Chapa, que hace parte de la cuenca hidrográfica del río Suárez, en la vereda San Pedro, del Municipio de Santana, Boyacá; está Localizada en las coordenadas 6 $02^{\prime} 85^{\prime \prime}$ 'Norte y $73^{\circ} 29^{\prime} 26^{\prime \prime}$ ' Oeste, a $1650 \mathrm{~m}$ de altitud. Debido a la actividad agrícola intensiva en la región, la cuenca se encuentra alterada, además, el caudal se ha modificado, por extracción de agua, para el abastecimiento de las veredas de la zona. $\mathrm{El}$ rango de temperatura media oscila entre 18 y $23^{\circ} \mathrm{C}$, con precipitación media multianual de $1700 \mathrm{~mm}$. Los periodos de lluvia, se concentran en mayo y octubre, con periodos de transición a meses de baja precipitación, en julio y en diciembre-enero. Santana tiene una estructura geomórfica de rocas sedimentarias, abundantes concreciones calco-piritosas y delgadas intercalaciones de calizas grasosas; en la fase arenosa hay alternancia de areniscas arcillosas de grano fino, con lutitas negras a amarillentas. Las formaciones vegetales que se pueden encontrar en Santana son fragmentos 
aislados de bosques húmedos subtropicales y bosques muy húmedos subtropicales (IGAC, 2005).

Diseño de muestreo: El trabajo, se llevó a cabo en dos épocas climáticas: lluvias, en octubre de 2010 y seca, en enero de 2011, con un muestreo cada una. Se seleccionaron tres puntos ubicados sobre un tramo de $300 \mathrm{~m}$, aproximadamente. Como criterios de selección, se consideraron: los sustratos de mayor frecuencia, como arena $(<2 \mathrm{~mm})$, grava $(2-16 \mathrm{~mm})$ y gravilla $(17-64 \mathrm{~mm})$, la ausencia de vertimientos puntuales entre los puntos, similitud en la condición de la fisonomía de la vegetación riparia, la cual, consistía en bosque subandino.

Variables físicas y químicas: Para caracterizar la condición física y química de la quebrada, se midió por punto: el $\mathrm{pH}$ y la temperatura, con $\mathrm{pH}$-metro Hanna; la conductividad eléctrica, con conductivímetro YSI 30; la concentración de Oxígeno y la saturación de Oxígeno, con el Oxímetro YSI y la alcalinidad y la dureza total, mediante el Kit Aqua-Merck colorimétrico. El Caudal, se estimó a través de la relación área de sección y velocidad de flujo (Gore, 2007):

$$
D=A \times v=\frac{w d a l}{t}
$$

Donde, $\mathbf{D}$ es la descarga o caudal, $\mathbf{A}$, el área de la sección transversal del río, $\mathbf{v}$, la velocidad del objeto flotante, $\mathbf{w}$, la anchura del lecho, d, la profundidad media del río, a, un coeficiente que varía de 0,8 , si el cauce es rugoso a 0,9 , si el cauce es liso, $\mathbf{l}$ es la distancia recorrida por el objeto y $\mathbf{t}$, el tiempo recorrido por el objeto.

Variables biológicas: Las muestras cualitativas, se tomaron con red de pantalla de $250 \mu \mathrm{m}$ de poro de malla. Para estimar la abundancia, se utilizó una red Surber, con un área de $900 \mathrm{~cm}^{2}$, con tres repeticiones aleatorias, en cada tramo. Los especímenes fueron preservados en alcohol, al 70\% (Wetzel \& Likens, 2000). La identificación taxonómica, se realizó a nivel de género, mediante las claves de Merritt et al. (2008) y Domínguez \& Fernández (2009).

La asociación de los grupos taxonómicos con los grupos funcionales alimenticios, se realizó por medio de información secundaria, tomada de Merritt et al. (2008), Tomanova et al. (2006) y Chará-Serna et al. (2010).

La biomasa, se halló mediante peso seco, que consistió en introducir los individuos separados por taxón, en el horno marca Memmert, a temperatura de $105^{\circ} \mathrm{C}$, durante cuatro horas y después pesarlos en una balanza analítica marca Ohaus Adventur Ar 1530-260, con sensibilidad de centésima de gramo; este método es sugerido por Wetzel \& Likens (2000) y Rodrigues-Capitulo et al. (2009).
Tratamiento de los datos: Para los datos físicos y químicos, se aplicó estadística descriptica; se estimó, promedio y coeficiente de variación, así como un análisis de componentes principales (PCA), para evaluar patrones de ordenación y variables determinantes; se llevó a cabo en el programa Past 3.1, versión libre.

Para conocer la estructura de los macroinvertebrados, se estimó la riqueza de géneros y se realizaron análisis gráficos, para la abundancia relativa y proporción porcentual de biomasa por taxón y época, considerando la clasificación de los grupos funcionales.

\section{RESULTADOS Y DISCUSIÓN}

Características físicas y químicas: El sistema presentó cambios temporales importantes en la expresión física y química. La conductividad eléctrica, el oxígeno disuelto, la alcalinidad y la dureza tienden a incrementarse en el periodo seco, contrario al comportamiento del caudal, la temperatura y el pH (Tabla 1). El caudal, la conductividad eléctrica y la alcalinidad expresaron la mayor variación temporal, con coeficientes de variación de 115, 48 y 46\%, respectivamente.

En el análisis de componentes principales, el primer componente explica el $70 \%$ y el segundo, el $22 \%$ de la variabilidad. El ordenamiento indica una distinción de los periodos hidroclimáticos, explicados, principalmente, por los cambios en la dureza, la alcalinidad y la conductividad eléctrica (Figura 1). Así, en el periodo de bajo caudal, hay concentración de iones en el sistema, entre ellos, los carbonatos, así como mayor concentración de oxígeno, lo que se interpretaría en la mayor productividad primaria (Sabater et al. 2009), en contraste a un proceso de dilución, que se evidencia en el periodo de mayor caudal, lo que es común en este tipo de ambientes (Allan \& Castillo, 2007).

Características biológicas: Los grupos taxonómicos hallados y la riqueza estimada en el estudio (Tabla 2), se encuentran, habitualmente, en sistemas de montaña andinos (Domínguez \& Fernández, 2009; Roldán et al. 2014; Vásquez-Ramos \& Reinoso, 2012). La mayor riqueza obtenida en época seca (26 taxones), se puede explicar por la mayor heterogeneidad espacial, disponibilidad de hábitats y recursos que, según Sabater et al. (2009) y Longo et al. (2010), es lo que sucede en momentos de reducción del caudal, lo que afecta, además, la distribución, la estructura, la composición y el estilo de vida de la comunidad.

En este sentido, la abundancia, la biomasa y la expresión de grupos funcionales en la comunidad también presentaron variación temporal, entre las épocas de bajas y altas lluvias; en la figura 2, se presenta la abundancia por taxón. Los géneros con mayor abundancia en la época de bajas lluvias 


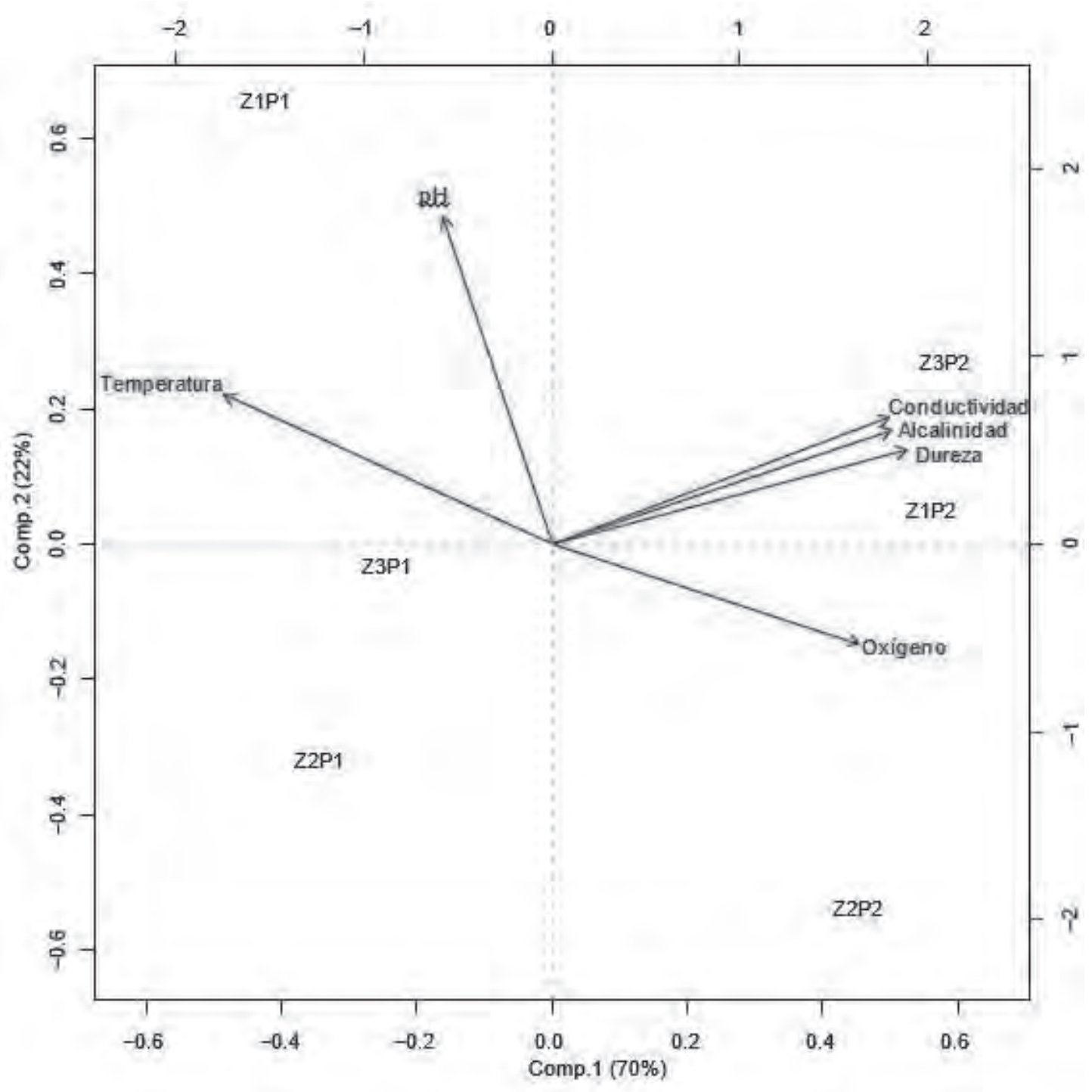

Figura 1. Análisis de componentes principales (ACP) de las variables físicas y químicas, en la quebrada La Chapa. Se indica la varianza explicada por cada componente. Z) zona, P1) periodo de lluvias altas y P2) periodo lluvias bajas.

Tabla 1. Variables físicas y químicas en la quebrada la Chapa.

\begin{tabular}{|c|c|c|c|c|c|c|c|c|c|c|}
\hline & Lluvias & $\mathrm{pH}$ & $\underset{{ }^{\circ} \mathrm{C}}{\text { Temp. }}$ & $\begin{array}{l}\text { Cond. } \\
\mu \text { S.cm }^{-1}\end{array}$ & $\underset{\mathrm{mg} . \mathrm{I}^{-1}}{\mathrm{OD}}$ & $\begin{array}{c}\text { Sat. } \\
\%\end{array}$ & $\begin{array}{l}\text { Alcal. } \\
\text { mg. } .^{1}\end{array}$ & Dureza & $\begin{array}{l}\text { Vel. } \\
\mathrm{m} . \mathrm{s}^{-1}\end{array}$ & $\begin{array}{l}\text { Caudal } \\
\mathrm{m}^{3} \cdot \mathrm{s}^{-1}\end{array}$ \\
\hline \multirow[t]{2}{*}{ Tramo 1} & Altas & 6,3 & 19,5 & 170 & 4,9 & 52,8 & 160 & 81 & 0,260 & 0,040 \\
\hline & Bajas & 6 & 18,7 & 320 & 7,5 & 77 & 320 & 175 & 0,032 & 0,006 \\
\hline \multirow[t]{2}{*}{ Tramo 2} & Altas & 5,9 & 19,1 & 98 & 5,2 & 55,9 & 110 & 61 & 0,620 & 0,720 \\
\hline & Bajas & 5,7 & 18,9 & 180 & 6,4 & 67,3 & 180 & 95 & 0,026 & 0,039 \\
\hline \multirow[t]{2}{*}{ Tramo 3} & Altas & 5,9 & 19,3 & 175 & 5,1 & 55,3 & 180 & 88 & SD & SD \\
\hline & Bajas & 5,9 & 18,8 & 410 & 6,04 & 64 & 420 & 182 & 0,064 & 0,040 \\
\hline
\end{tabular}


Tabla 2. Composición de la comunidad de macroinvertebrados, de la quebrada La Chapa. Se indica el grupo funcional alimenticio, al cual, pertenece cada taxón.

\begin{tabular}{|c|c|c|c|c|}
\hline \multirow{2}{*}{ Familia } & \multirow{2}{*}{ Taxón específico } & \multirow{2}{*}{ Grupo funcional } & \multicolumn{2}{|c|}{ Época } \\
\hline & & & A & B \\
\hline Naididae & Naididae & Colector-recolector & 1 & 1 \\
\hline Asellidae & Asellidae & Colector-recolector & 0 & 1 \\
\hline \multirow[t]{4}{*}{ Baetidae } & Andesiops sp. & Colector-recolector & 1 & 0 \\
\hline & Baetodes sp. & Raspador & 0 & 1 \\
\hline & Prebaetodes sp. & Colector-recolector & 0 & 1 \\
\hline & Mayobaetis sp. & Colector-recolector & 0 & 1 \\
\hline Leptohyphidae & Leptohyphes sp. & Colector-recolector & 1 & 1 \\
\hline \multirow[t]{3}{*}{ Leptophlebiidae } & Thraulodes sp. & Colector-recolector & 1 & 1 \\
\hline & Ecuaphlebia sp. & Raspador & 1 & 1 \\
\hline & Traverella sp. & Colector-recolector & 1 & 0 \\
\hline Perlidae & Anacroneuria sp. & Depredador & 0 & 1 \\
\hline Calopterygidae & Hetaerina sp. & Depredador & 0 & 1 \\
\hline Coenagrionidae & Argia sp. & Depredador & 1 & 0 \\
\hline Libellulidae & Libellula sp. & Depredador & 1 & 0 \\
\hline Naucoridae & Limnocoris sp. & Perforador-carnívoro & 0 & 1 \\
\hline Veliidae & Rhagovelia sp. & Perforador-carnívoro & 1 & 1 \\
\hline \multirow[t]{2}{*}{ Elmidae } & Cylloepus sp. & Colector-recolector & 0 & 1 \\
\hline & Disersus sp. & Colector-recolector & 0 & 1 \\
\hline Ptilodactylidae & Anchytarsus sp. & Fragmentador & 1 & 1 \\
\hline Helicopsychidae & Helicopsyche sp. & Raspador & 0 & 1 \\
\hline \multirow[t]{2}{*}{ Hydroptilidae } & Metrichia sp. & Raspador & 0 & 1 \\
\hline & Hydroptila sp. & Raspador & 0 & 1 \\
\hline \multirow[t]{3}{*}{ Hydropsychidae } & Leptonema sp. & Fragmentador & 0 & 1 \\
\hline & Hydropsychidae & Fragmentador & 1 & 0 \\
\hline & Smicridea sp. & Fragmentador & 0 & 1 \\
\hline Polycentropodidae & Cyrnellus sp. & Depredador & 0 & 1 \\
\hline Odontoceridae & Odontoceridae & Colector-recolector & 0 & 1 \\
\hline Pyralidae & Pyralidae & Fragmentador & 1 & 0 \\
\hline Ceratopogonidae & Ceratopogonidae & Depredador & 1 & 0 \\
\hline \multirow[t]{2}{*}{ Chironomidae } & Parametriocnemus sp. & Colector-recolector & 0 & 1 \\
\hline & Lopescladius sp. & Colector-recolector & 0 & 1 \\
\hline \multirow[t]{2}{*}{ Simuliidae } & Simulium sp. 1 & Colector-filtrador & 1 & 1 \\
\hline & Simulium sp. 2 & Colector-filtrador & 1 & 0 \\
\hline Tipulidae & Tipulidae & Colector-recolector & 1 & 0 \\
\hline Arrenuridae & Arrenuridae & Perforador-carnívoro & 0 & 1 \\
\hline Hydrobiidae & Hydrobiidae & Raspador & 0 & 1 \\
\hline \multicolumn{3}{|c|}{ Total } & 16 & 26 \\
\hline \multicolumn{3}{|c|}{ Índice de similitud de Jaccard (coeficiente de similitud Ij) } & \multicolumn{2}{|c|}{0,2} \\
\hline
\end{tabular}


fueron Thraulodes sp. (37 individuos) y Helicopsyche sp. (85 individuos), ya que ambientes con corriente moderada, se consideran su hábitat preferente (Vásquez-Ramos \& Reinoso, 2012). Por otro lado, en la época de lluvias, Simulium sp. (7 individuos), fue el grupo dominante, dado que prefieren flujos de agua continua y de mayor velocidad (GallardoMayenco \& Toja, 2002).
En contraste con la abundancia, los grupos dominantes en biomasa, para la época seca, fueron el hemíptero Limnocoris sp. $\left(0,16 \mathrm{~g} / \mathrm{m}^{2}\right)$ y el gasterópodo de la familia Hydrobiidae $\left(0,059 \mathrm{~g} / \mathrm{m}^{2}\right)$, mientras que en la época de lluvias, fueron los lepidópteros de la familia Pyralidae $\left(0,29 \mathrm{~g} / \mathrm{m}^{2}\right)$ (Figura 3). El contraste entre estos descriptores cuantitativos, se debe a que la biomasa está afectada, en mayor grado, por el tamaño corporal que por la abundancia (Peters, 1983).

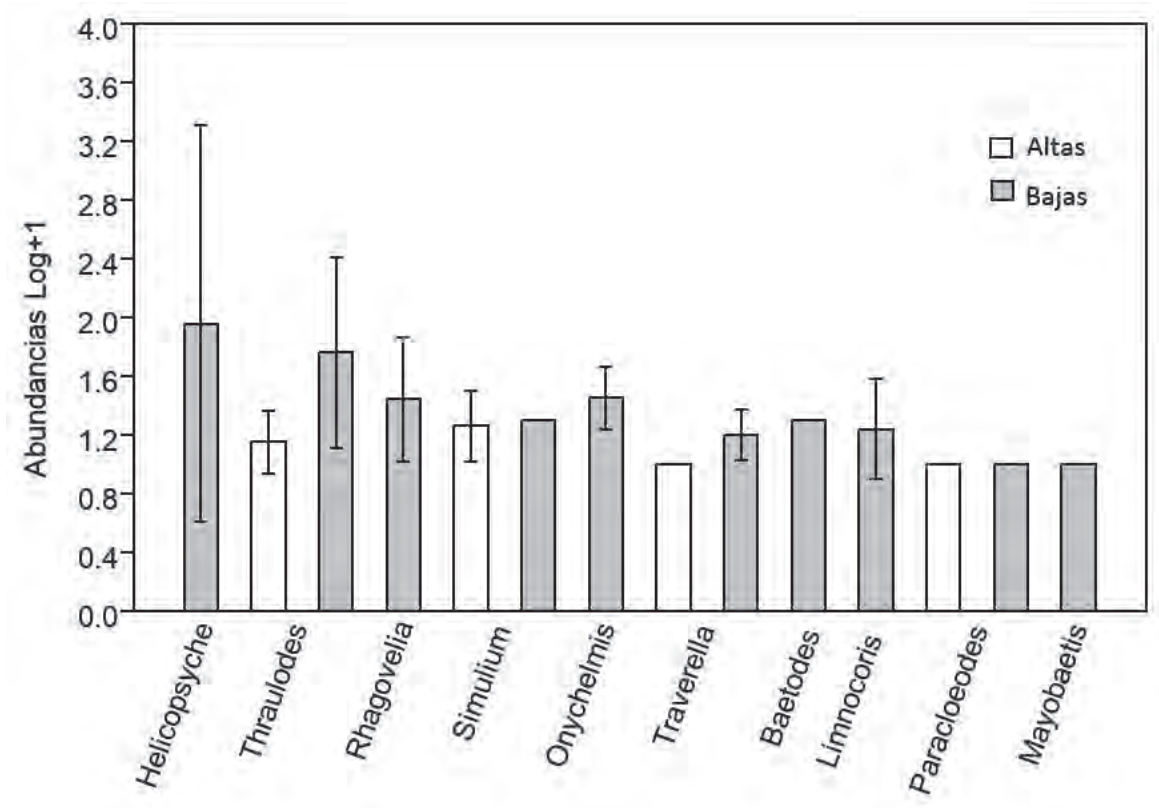

Figura 2. Abundancia transformada Log +1 de los taxones encontrados en la quebrada la Chapa, en los periodos de lluvias altas y bajas.

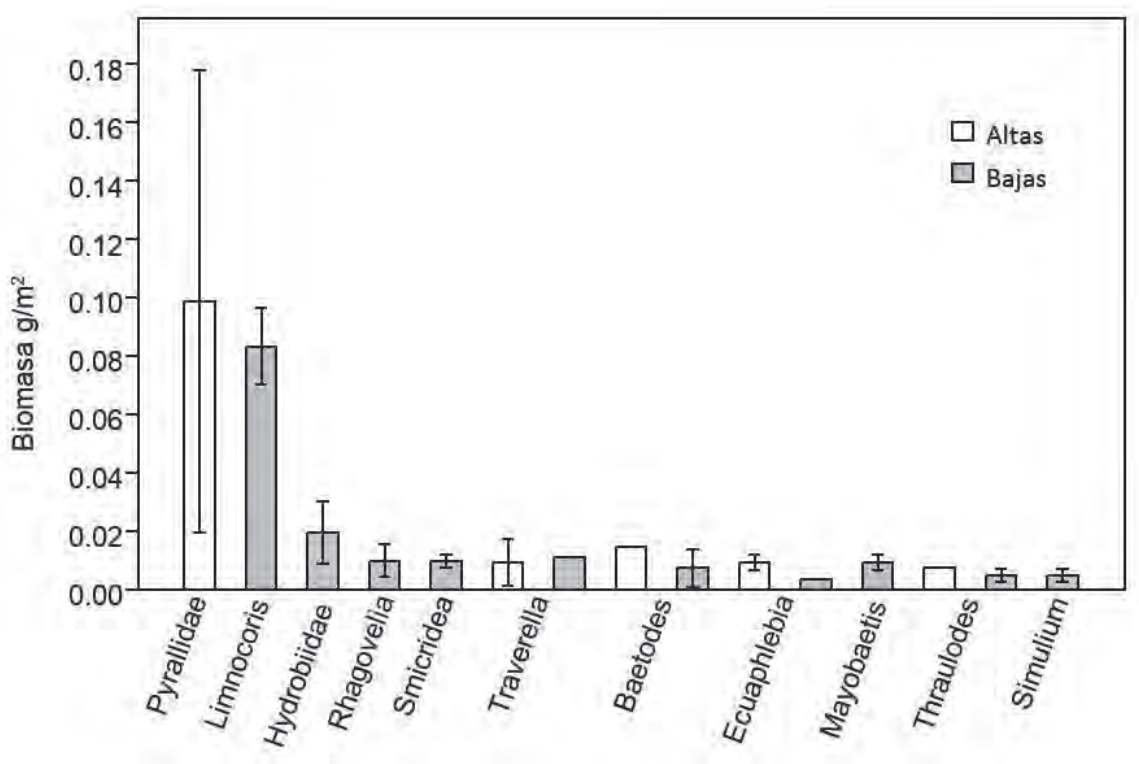

Figura 3. Biomasa de los taxones encontrados en la quebrada la Chapa, en periodo lluvias altas y bajas. 
Grupos funcionales alimenticios: En cuanto a los grupos funcionales alimenticios encontrados, se observó gran variación temporal. Esta condición, se puede dar en función de los cambios del caudal, sustrato y al efecto que pueda tener la vegetación ribereña dentro del sistema, como fuente aportante de recursos, ya que la entrada alóctona y detritus son la principal fuente de energía para muchos de los organismos que habitan los ríos, en las zonas de cabecera (Jacobsen, 2008; Uieda \& Motta, 2007; Lorion \& Kennedy, 2009).

En la época seca hubo mayor abundancia de organismos raspadores (Figura 4), que se relacionan con la mayor dis- ponibilidad de perifiton en la quebrada que, según Biggs \& Close (1989), se incrementa en condiciones de corriente moderada a baja. La mayor concentración de oxígeno estimada para este momento sería indicador del incremento de la actividad de las algas del fito-perifiton (Sabater et al. 2009). En contraste en el periodo de lluvias, los grupos con mayor abundancia fueron colectores-recolectores y colectores-filtradores, que se vinculan con el aumento del transporte de MOPF, lo que es habitual en época de precipitaciones, en este tipo de sistemas (Uieda \& Motta, 2007; Wantzen et al. 2008; Tamarís-Turizo \& Rodríguez-Barrios, 2015).

\section{Abundancia}

Lluvias altas

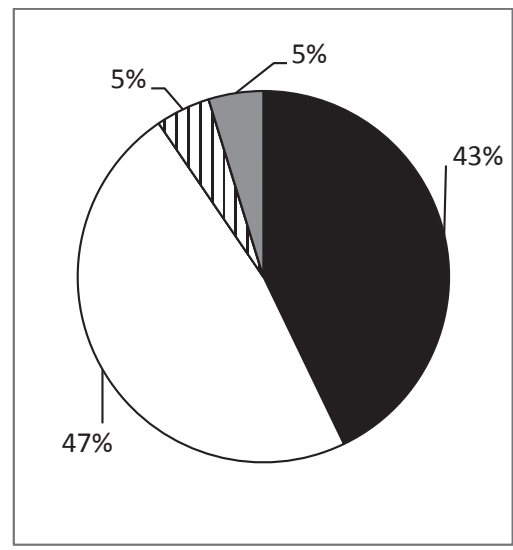

Lluvias bajas

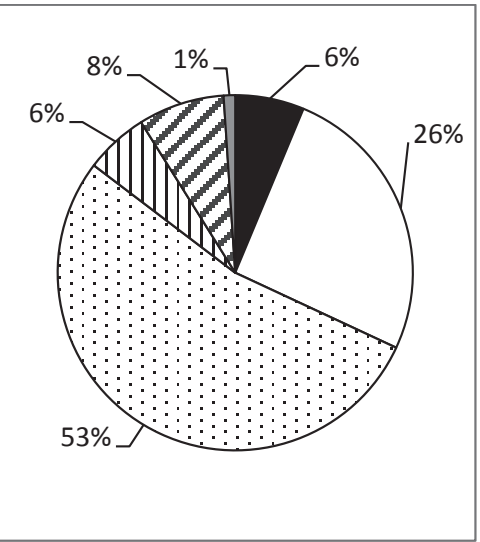

Biomasa

Lluvias altas

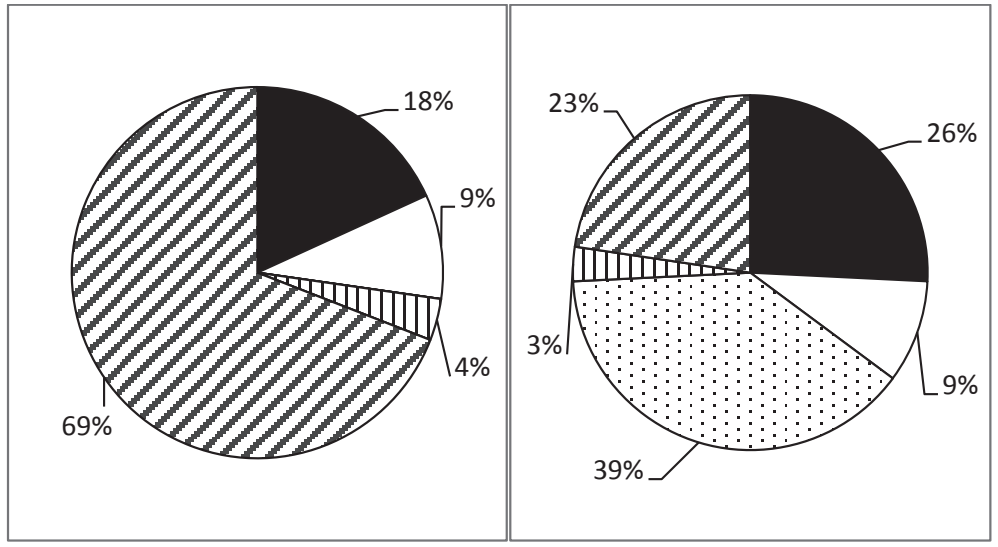

Lluvias bajas

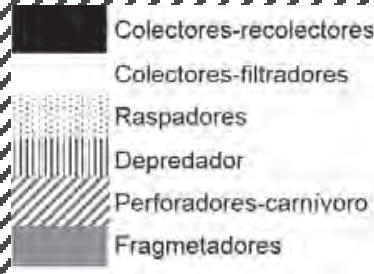

Figura 4. Proporción de grupos funcionales alimenticios para abundancia y biomasa, en época de lluvias altas y bajas, en la quebrada la Chapa. 
Respecto al comportamiento de los grupos funcionales, medida en biomasa (Figura 4), en la época seca, los organismos raspadores $\left(0,18 \mathrm{~g} / \mathrm{m}^{2}\right)$ y colectores-recolectores $\left(0,12 \mathrm{~g} / \mathrm{m}^{2}\right)$ tuvieron mayor biomasa, mientras que en época de lluvias, los organismos con mayor biomasa fueron los perforadorescarnívoros $\left(0,14 \mathrm{~g} / \mathrm{m}^{2}\right)$ y ello se explica, por el efecto del mayor tamaño de estos organismos (Margalef, 1983; Príncipe et al. 2010; Smock et al. 1985).

Como conclusión, se confirma que los cambios temporales asociados con la estacionalidad climática e hidráulica son importantes en la estructuración de las comunidades de macroinvertebrados, de las quebradas de montaña.

La interpretación de la abundancia y de la biomasa de los macroinvertebrados, como descriptores cuantitativos de estructura y de funcionalidad de un sistema de este tipo, varía; esto, en función de la morfología y el hábito trófico de los organismos; sin embargo, rasgos alimenticios, como raspador y colector-recolector, independientemente del descriptor, fueron representativos.

La expresión de los grupos funcionales alimenticios en arroyos de montaña está asociada a la oferta de recursos y el tipo de metabolismo del sistema, por tanto, podría ser un buen criterio de indicación de su calidad ecológica, como complemento a los sistemas de evaluación, basados en composición y en estructura.

Agradecimientos: Al grupo de investigación UDESA de la UPTC, por su constante apoyo en el desarrollo de la investigación. A los biólogos Karen Lizeth Pulido, Alcybiades Escarraga y Luz Nidia Gil, por el apoyo en la primera fase del proyecto. Al doctor Jonathan Shurin, por la revisión idiomática del abstract. A los revisores anónimos, por los valiosos aportes a la cualificación del manuscrito. Conflictos de intereses: El manuscrito fue preparado y revisado con la participación de los autores, quienes declaramos que no existe conflicto de intereses, que ponga en riesgo la validez de los resultados presentados.

\section{BIBLIOGRAFÍA}

1. ALLAN, D.; CASTILLO, M. 2007. Stream Ecology. Structure and function of running waters.Ed. Springer (Netherlands). 436p.

2. BIGGS, B.; CLOSE, M. 1989. Periphyton biomass dynamics in gravel bed rivers: the relative effects of flows and nutrients. Freshwater Biology. (USA). 22(2):209231.

3. BONADA, N.; PRAT, N.; RESH, V.; STATZNER, B. 2006. Developments in aquatic insect biomonitoring: $A$ comparative analysis of recent approaches. Ann. Rev. Entomol. (Brasil). 51:495-523.

4. CHARÁ-SERNA, A.M.; CHARÁ, J.D; ZÚĨ̃IGA, M. del C.; PEDRAZA, G.X.; GIRALDO, L.P. 2010. Clasificación trófica de insectos acuáticos en ocho quebradas protegidas de la ecorregión cafetera colombiana. Universitas Scientiarum. 15(1):27-36.

5. CUMMINS, K. 1974. Structure and function of stream ecosystem. BioScience. (USA). 24 (11):631-641.

6. DOMÍNGUEZ, E.; FERNÁNDEZ, H. 2009. Macroinvertebrados bentónicos sudamericanos: sistemática y biología. Ed. Fundación Miguel Lillo (Argentina). 654p.

7. GALLARDO-MAYENCO, A.; TOJA, J. 2002. Spatiotemporal distribution of Simuliids (Diptera) and associated environmental factors in two mediterranean basins of Southern Spain. Limnetica. (España). 21(1-2):47-57.

8. GARCÍA, M.; VARGAS, O.; SANTOS, A.; BERNAL, F.; OLAYA, G.; ROSERO, M.; CEBALLOS, J.; GONZALEZ, J.; ALFONSO, N.; GARZON, C.; CAMPILLO, A.; ONOFRE, C.; AREVALO, D.; CAMPUZANO, C.; RODRIGUEZ, C.; GONZALEZ, J.; GUZMAN, A.; PARADA, G.; ZARATE, E.; TETAY, C.; ORJUELA, L.; CARDENAS, O.; DIAZ, D.; CASTAÑEDA, C.; BELTRAN, M.; MONTOYA, J.; CONTRERAS, C.; CAICEDO, F.; AGUIRRE, S. 2015. Estudio nacional del agua IDEAM. Ed. Panamericana formas e impresos. (Colombia). 493p.

9. GORE, J. 2007. Discharge measurements and streamflow analysis. En: Hauer, F.; Lamberti, G. (eds). Methods in Stream Ecology. Ed. Elsevier Science (USA). p.51-78.

10. GUZMÁN-SOTO, C.; TAMARÍS-TURIZO, C. 2014. Hábitos alimentarios de individuos inmaduros de Ephemeroptera, Plecoptera y Trichoptera en la parte media de un río tropical de montaña. Rev. Biol. Trop. (Costa Rica). 62(2):169-178.

11. INSTITUTO GEOGRÁFICO AGUSTÍN CODAZZI -IGAC-. 2005. Estudio General de Suelos y Zonificación de Tierras del Departamento de Boyacá, Tomo I.

12. JACOBSEN, D. 2008. Tropical high-altitude streams. En: Dudgeon, D. (ed). Tropical Stream Ecology. Ed. Elsevier Science (USA). p.219-253. 
13. LONGO, M.; ZAMORA, H.; GUISANDE, C.; RAMÍREZ, J. 2010. Dinámica de la comunidad de macroinvertebrados en la quebrada Potrerillos (Colombia): Respuesta a los cambios estacionales de caudal. Limnetica. (España). 29(2):195-210.

14. LORION, C.; KENNEDY, B. 2009. Relations between deforestation, Riparian forest buffers and benthic macroinvertebrates in neotropical head water streams. Freshwater Biology. (USA). 54: 165-180.

15. MARGALEF, R. 1983. Limnología. Ed. Omega, S.A. (España).1010p.

16. MERRITT, R.; CUMMINS, K.; BERG, M. 2008. An Introduction to the Aquatic Insects of North America. Ed. Kendall/Hunt Company. (USA). 1158p.

17. PETERS, R. 1983. The Ecological Implications of Body Size. Ed. Cambridge University Press (Reino Unido). 329p.

18. PRÍNCIPE, R.; GUALDONI, C.; OBERTO, A.; RAFFAINI, G.; CORIGLIANO, M. 2010. Spatial-temporal patterns of functional feeding groups in mountain streams of Córdoba. Ecología Austral. (Argentina). 20:257-268.

19. RIOS-TOUMA, B.; ENCALADA, A.C.; PRAT, N. 2011. Macroinvertebrate assemblages of an Andean highaltitude tropical stream: the importance of season and flow. Int. Rev. Hydrobiol. (USA). 96(6):667-685.

20. RIVERA, J.; PINILLA, G.; CAMACHO, D. 2013. Macroinvertebrate Trophic Groups in an Andean Wetland of Colombia. Acta Biol. Col. 18(2):279-292.

21. RODRIGUES-CAPITULO, A.; MUÑOZ, I.; BONADA, N.; GAUDES, A.; TOMANOVA, S. 2009. La biota de los ríos: los invertebrados. En: Elosegi, A.; Sabater, S. (eds.). Conceptos y técnicas en ecología fluvial. Ed. Fundación BBVA. (España). p.253-270.

22. RODRÍGUEZ-BARRIOS, J.; OSPINA-TÓRRES, R.; TURIZO-CORREA, R. 2011. Grupos funcionales alimentarios de macroinvertebrados acuáticos en el río Gaira, Colombia. Rev. Biol. Trop. (Costa Rica).59(4):15371552.
23. ROLDÁN, G.; ZÚINIGA, M.; ZAMORA, L.; REINOSO, G.; LONGO, M. 2014. Colombia. En: Alonso-Eguía Lis, P.; Mora, J.; Campbell, B.; Springer, M. (eds). Diversidad, conservación y uso de los macroinvertebrados dulceacuícolas de México, Centroamérica, Colombia, Cuba y Puerto Rico. Ed. D.R. Instituto Mexicano de Tecnología del Agua. (México). p.63-93.

24. SABATER, S.; DONATO, J.; GIORGI, A.; ELOSEGI, A. 2009. El río como ecosistema. En: Elosegi, A.; Sabater, S. (eds). Conceptos y técnicas en ecología fluvial. Ed. Fundación BBVA. (España). p.23-37.

25. SMOCK, L.; GILINSKY, E.; STONEBURNER, D. 1985. Macroinvertebrates production in southeastern United States blackwater streams. Ecology. (USA). (66):1491-1503.

26. TAMARÍS-TURIZO, C.; RODRÍGUEZ-BARRIOS, J. 2015. Transport of organic matter across a tropical mountain stream of Sierra Nevada de Santa Marta (Colombia). Acta Biol. Col. 20(3):209-216.

27. TOMANOVA, S.; GOITIA, E.; HELEŠIC, J. 2006. Trophic levels and functional feeding groups of macroinvertebrates in neotropical streams. Hydrobiologia. (Alemania). 556:251-264.

28. UIEDA, V.; MOTTA, R. 2007. Trophic organization and food web structure of southeastern Brazilian streams: A review. Acta Limnol. Bras. 19(1):15-30.

29. VÁSQUEZ-RAMOS, J.; REINOSO, G. 2012. Estructura de la fauna béntica en corrientes de los Andes colombianos. Rev. Col. Entomol. 38(2):351-358.

30. WANTZEN, K.; YULE, C.; MATHOOKO, J.; PRINGLE, C. 2008. Organic Matter Processing in Tropical Streams. En: Dudgeon, D. (ed.). Tropical Stream Ecology. Ed. Elsevier Science (USA). p.43-64.

31. WETZEL, R.; LIKENS, G. 2000. Limnological Analyses. Springer-Verlag. (USA). 429p.

Recibido: Mayo 6 de 2016

Aceptado: Octubre 24 de 2016

Cómo citar:

Motta Díaz, A.; Ortega Corredor, L.; Niño Fernández, Y.; Aranguren Riaño, N. 2016. Grupos funcionales alimenticios de macroinvertebrados acuáticos en un arroyo tropical (Colombia). Rev. U.D.C.A Act. \& Div. Cient. 19(2): 425-433. 